\title{
Une syringocèle révélée par un abcès périnéal
}

\author{
Kamel CHABCHOUB, Hussein KANDIL, Firas ZAHWA, Philippe DANJOU
}

Centre hospitalier Dr Schaffner, Lens

\section{RÉSUMÉ}

Le terme syringocèle désigne la dilatation kystique des canaux des glandes de Cowper. Nous rapportons le premier cas de syringocèle découverte à l'occasion d'un abcès périnéal étendu chez un homme âgé de 50 ans.

Le diagnostic a été confirmé par le scanner, l'urétrographie rétrograde et l'urétroscopie. Le traitement a consisté en un drainage chirurgical par voie périnéale associée à une dérivation urinaire et une antibiothérapie adaptée.

Le but de ce travail est de discuter les circonstances de découverte ainsi que les modalités diagnostiques et thérapeutiques de cette pathologie peu fréquente.

Mots clés : syringocèle, glandes de Cowper, abcès

\section{INTRODUCTION}

La syringocèle, pathologie peu fréquente, est une dilatation congénitale ou acquise des canaux des glandes de Cowper.

Sur le plan anatomique, les canaux et les glandes de Cowper sont réparties en deux paires. Une paire de glandes principales diaphragmatiques à laquelle s'associe une autre paire accessoire enfouie au sein du corps spongieux, de part et d'autre de l'urètre bulbaire $[3,10]$. Les glandes principales sont situées entre les feuillets superficiel et profond de l'aponévrose périnéale moyenne, au sein du muscle transverse profond dont la contraction en exprime le contenu.
Elles mesurent en moyenne $10 \mathrm{~mm}$ de long, 4,5 mm de haut et $6,5 \mathrm{~mm}$ de large [11].

C'est la connaissance de cette anatomie qui permet de mieux comprendre les images radiologiques et les pathologies correspondantes (Figure 1).

\section{OBSERVATION}

Monsieur D. F., âgé de 50 ans, sans antécédents pathologiques, a été hospitalisé en urgence pour abcès périnéal. Une intoxication tabagique à 30 paquetsannée avec consommation alcoolique non négligeable ont été notées à l'interrogatoire.

L'histoire de la maladie a débuté une semaine avant par des brûlures mictionnelles associées à une fièvre, frisson et douleur périnéale. L'examen clinique a mis en évidence une tuméfaction périnéale rénitente, inflammatoire et douloureuse, se prolongeant à la verge sans atteinte du scrotum. Au toucher rectal, la prostate était souple, indolore, non abcédée, avec douleur exquise à la hauteur du plancher pelvien au dessous du bec prostatique.

A la biologie, le syndrome inflammatoire était évident, sans insuffisance rénale ni élévation du taux de PSA $(0,47 \mathrm{ng} / \mathrm{ml})$.

Au scanner abdominopelvien, le plancher périnéal était le siège d'une collection abcédée se projetant en regard de l'urètre bulbaire (Figure 2), et se prolongeant en haut vers les glandes de Cowper (Figure 3), et en bas vers la verge dont le fourreau a été décollé (Figure 4).

Correspondance :

Dr Kamel CHABCHOUB - Service urologie, CH de Lens, 99 route de la Bassée SP 8, 62307 Lens - Tel 0661205003

- 0321691570 - Fax 0321691575 -

Email k.chabchoub@laposte.net 
Une triple antibiothérapie à large spectre, associant une béta-lactamine, un aminoside et un imidazole, a été aussitôt instaurée, et un drainage chirurgical de l'abcès par voie périnéale a été réalisé en urgence, associé à la pose d'un cathéter sus pubien. L'évolution a été marquée par la persistance d'une fièvre oscillante en rapport avec la persistance d'une collection résiduelle objectivée sur le scanner de contrôle (Figure 5). Ceci a nécessité un second drainage avec décapage et débridement sous anesthésie générale. Le pansement a été changé par la suite quotidiennement deux fois par jours. L'apyrexie a été obtenue rapidement avec amélioration de l'état général.

L'analyse bactériologique des urines, des hémocultures ainsi que le pus prélevé ont été positives à Echérichia Coli sensible à tous les antibiotiques. L'antibiothérapie a été adaptée secondairement en fonction de ces résultats.

A l'urétrocystographie réalisée après traitement de l'infection, les canaux et les glandes de Cowper surmontant un urètre bulbaire diverticulaire, ont été opacifiés (Figure 6). L'urétroscopie a montré les orifices de perforation au sein d'une boursouflure effondrée, correspondant à la syringocèle perforée au niveau de l'urètre bulbaire, sans rétrécissement urétral associé (Figure 7). La cystoscopie a été normale.

Après un recul d'un mois, le patient urine bien, sans dysurie ni fuites urinaires.

\section{DISCUSSION}

La syringocèle congénitale concerne la population pédiatrique [4], tandis que la forme acquise survient chez l'homme plus âgé, et le plus souvent secondairement à une obstruction post infectieuse [5]. Ceci est le cas pour notre patient chez qui a été découvert des calcifications prostatiques secondaire probablement à des épisodes infectieux.

Les manifestations cliniques des syringocèles ne sont pas spécifiques. II peut s'agir de douleur périnéale, notamment en cas d'atteinte des glandes diaphragmatiques, ou d'une symptomatologie liée au bas appareil urinaire telle qu'une hématurie, une dysurie, une incontinence urinaire post mictionnelle ou même une infection urinaire $[1,6,7,9,12,14-16]$. A notre connaissance, cette observation rapporte le premier cas de syringocèle révélée par un abcès périnéal étendu.

La confirmation du diagnostic est radiologique et endoscopique. Le principal examen radiologique qui permet de faire le diagnostic de la syringocèle est l'urétrocystographie $[11,6,8]$. L'aspect radiologique de la syringocèle en urétrocystographie est représenté par une image d'addition sous le plancher urétral [11]. Cet aspect rentre dans le cadre d'une classification radiologique décrite par Maizels et al. [9]. Cette classification comporte quatre stades variant en fonction du degré de communication de la syringocèle avec l'urètre (Figure 8) [9]. D'autres critères radiologiques plus spécifiques de la dilatation kystique de la glande de Cowper sont décrits : le parallélisme de l'image à l'urètre, la limite supérieure ne dépassant pas le diaphragme uro-génital, la configuration souvent tubulaire, la position de l'orifice sur la face ventrale de l'urètre bulbaire [2].

L'IRM pelvienne permet de faire le diagnostic lorsque l'urétrocystographie est non concluante [7]. L'aspect de la syringocèle en IRM est celui d'une image kystique ovalaire homogène, développée au dépens du plancher de l'urètre bulbaire dont la lumière est légèrement comprimée [7].

L'examen endoscopique retrouve une boursouflure translucide de couleur grise ou bleue, sphérique, sur le plancher de l'urètre bulbaire en cas de syringocèle non perforée [11].

Dans notre cas l'aspect et la topographie de l'abcès au scanner sont évocateurs. L'urétrocystographie et l'urétroscopie ont confirmé l'aspect perforé de la syringocèle. Ce dernier est classé type $C$ selon la classification de Maizels et al. [9].

La prise en charge thérapeutique de la syringocèle est le plus souvent endoscopique. Elle consiste en la marsupialisation endoscopique de la dilatation kystique à l'anse électrique ou au laser Holmium YAG $[1,6,7$, $9,12]$.

En cas de syringocèle à manifestation périnéale, une cure chirurgicale par voie périnéale avec urétroplastie dans le même temps est proposée [13].

Notre patient a eu un drainage de sa syringocèle abcédée par voie périnéale, associé à une dérivation urinaire qui a été gardée trois semaines. L'urétrographie réalisée au bout de cette période n'a pas objectivé de fistule urétrale. Al'examen endoscopique, la syringocèle est bien drainée. Aucun autre geste opératoire n'a été réalisé.

\section{CONCLUSION}

La syringocèle est une pathologie peu fréquente. Son diagnostic clinique est difficile. Les examens radiologiques et endoscopiques permettent de rétablir le diagnostic. La marsupialisation endoscopique constitue le traitement des formes simples non compliquées, tandis que la chirurgie ouverte par voie périnéale est réservée pour les formes compliquées. 


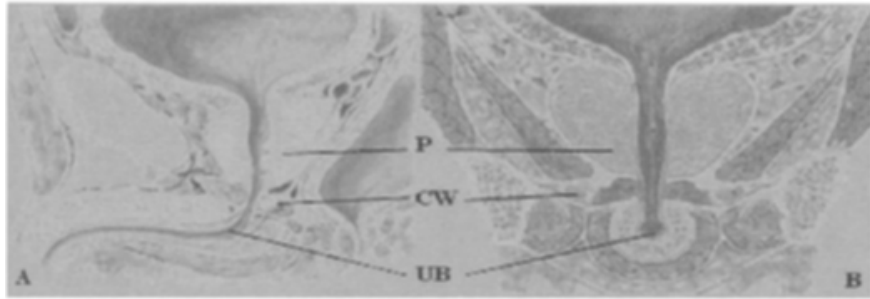

Figure 1 : Coupes anatomiques du pelvis masculin : A : coupe sagittale $B$ : coupe frontale. $(P)$ prostate, $(C W)$ glandes de Cowper, (UB) urètre bulbaire.

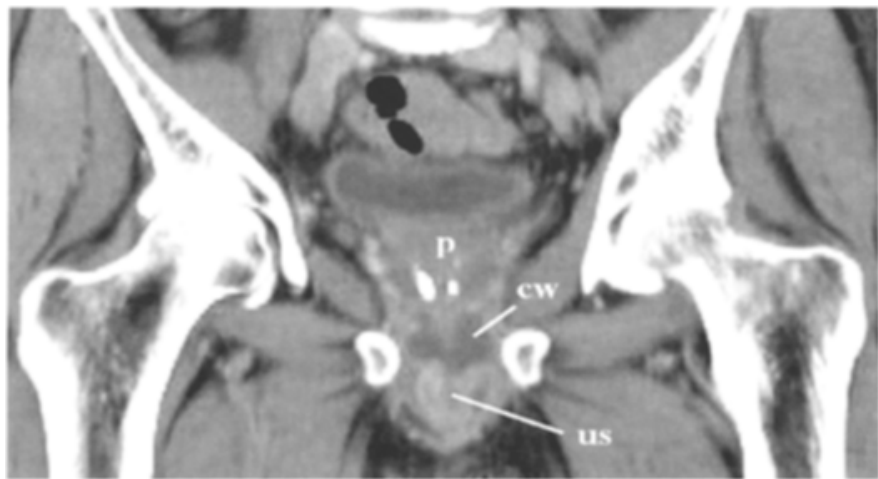

Figure 3 : La prostate (p) est le siège de calcifications. Elle surmonte séparément les glandes de Cowper (cw) dilatées et infectées. Ces glandes sont en communication avec l'urètre spongieux (us).

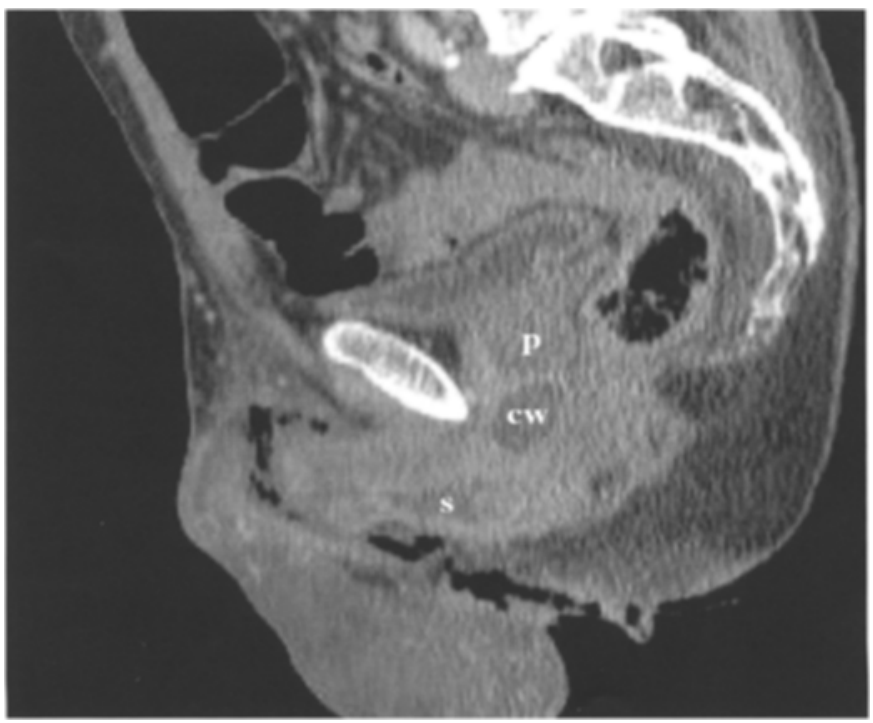

Figure 5 : Coupe sagittale du pelvis au scanner de contrôle : Collection résiduelle infectée au sain des glandes de Cowper (cw). (s) Syringocèle se projetant en regard du bulbe urétral. (p) Prostate.

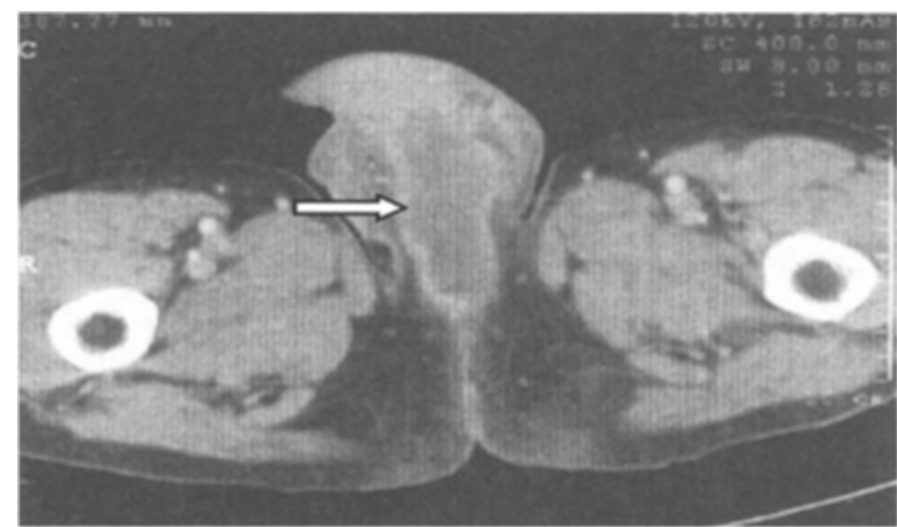

Figure 2 : Collection abcédée en regard du bulbe urétral.

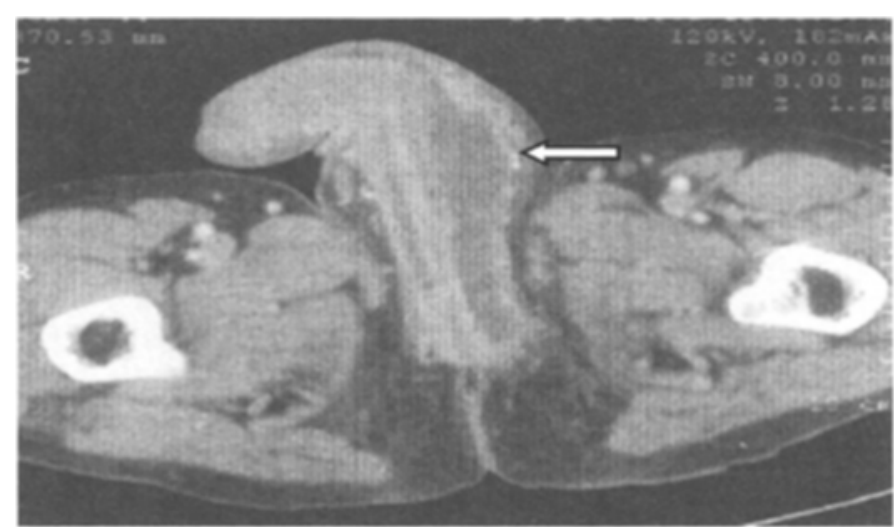

Figure 4 : Décollement du fourreau de la verge par l'abcès.

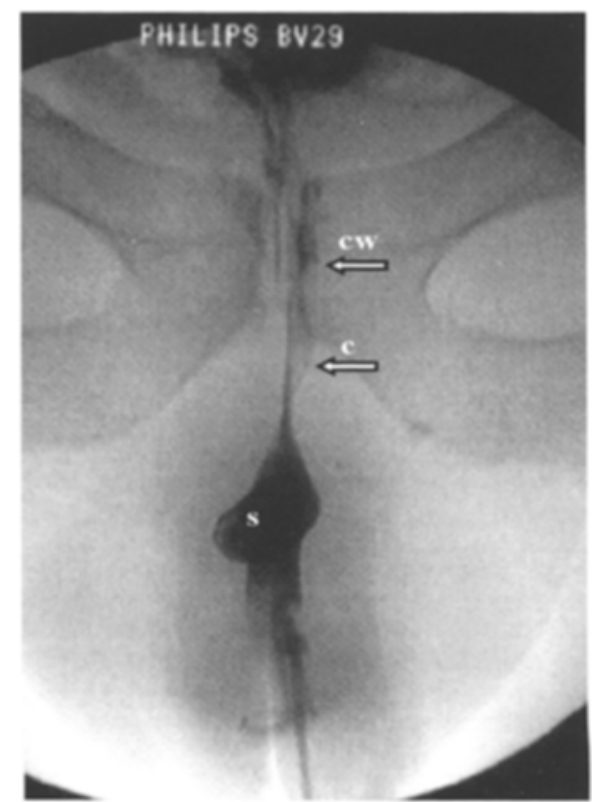

Figure 6 : Urétrocystographie rétrograde vue de face : (s) syringocèle. Opacification des conduits de Cowper (c) qui sont parallèles à l'urètre ainsi que des glandes de Cowper (cw) dilatées. 


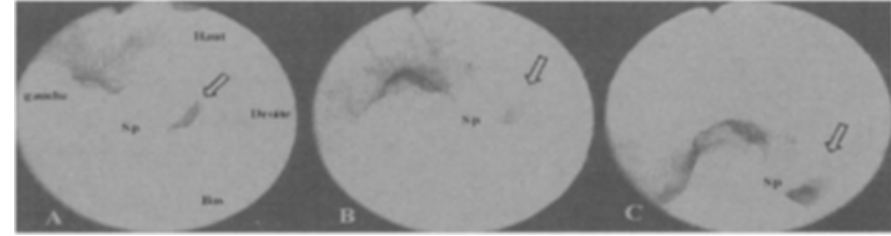

Figure 7 : Urétroscopie : Aspect de la syringocèle perforée (sp) à plusieurs endroits de l'urètre bulbaire (flèches). A : partie distale de l'urètre bulbaire ; $B$ : partie moyenne ; $C$ : partie proximale. $(L U)$ lumière urétrale.

\section{RÉFÉRENCES}

1. BRANDES S.B., R.E : Cowper's syringocele : symptoms, classification and treatment of an unappreciated problem. J. Urol., 2000, 164 : 1666-1668.

2. BROCK W.A., KAPLAN G.W. : Lesions of Cowper 's glands in children. J. Urol., 1979, 122 : 121-123.

3. CADY J., KRON B. : Anatomie du corps humain. Paris, Maloine, 1970 , fasc. $5: 108$.

4. CAMPOBASSO P., SCHIEVEN E., FERNANDES E.C. : Cowper's synringocele : an analysis of 15 consecutive cases. Arch. Dis. Child., 1996, $75:$ 71-73.

5. COLODNY A.H., LEBOWITZ R.L. : Lesions of Cowper's ducts and glands in infants and children. Urology, 1978, 11 : 321-325.

6. GHADOUANE M., ALAMI M., KASMAOUI E.H., OUHBI Y., SAFI L. : Abbar M. : Syringocèle : à propos de 2 cas. Prog. Urol., 2001, 11 : 354-356.

7. KICKUTH R., LAUFER U., PANNEK J., KIRCHNER T.H., HERBE E., KIRCHNER J. : Cowper's syringocele : diagnosis based on MRI findings. Pediatr. Radiol, 2002, 32 : 56-58.

8. LEBOWITZ R.L. : Letter to the editor «Cowper's gland duct: radiologic finding». Urol. Radiol., 1992, $13: 200$.

9. MAIZELS M., STEPHENS F., KING L.R., FIRLIT C.F. : Cowper's synringocele : a classification of dilatation of Cowper's gland duct based upon clinical characteristics of 8 boys. J. Urol., 1983, 129, 111-114.

10. MASSON J.C., SUHLER A., GARBAY B. : Les canaux et glandes de Cowper : manifestations pathologiques et aspects radiologiques. J. Urol. Nephrol., 1979, $85:$ :997-511.

11. PEDRON P., TRAXER O., HAAB F. et al. : Glandes de Cowper: aspects anatomique, physiologique et pathologique. Prog. Urol., 1997, $7:$ 563-569.

12. PIEDRAHITA Y.K., PALMER J.S. : Case report : Cowper's syringocele treated with Holmium : YAG laser. J. Endourol., 2006, $20: 677-678$.

13. REDMAN J.F., ROUNTREE G.A. : Pronounced dilatation of Cowper's gland duct manifest as perineal mass : a

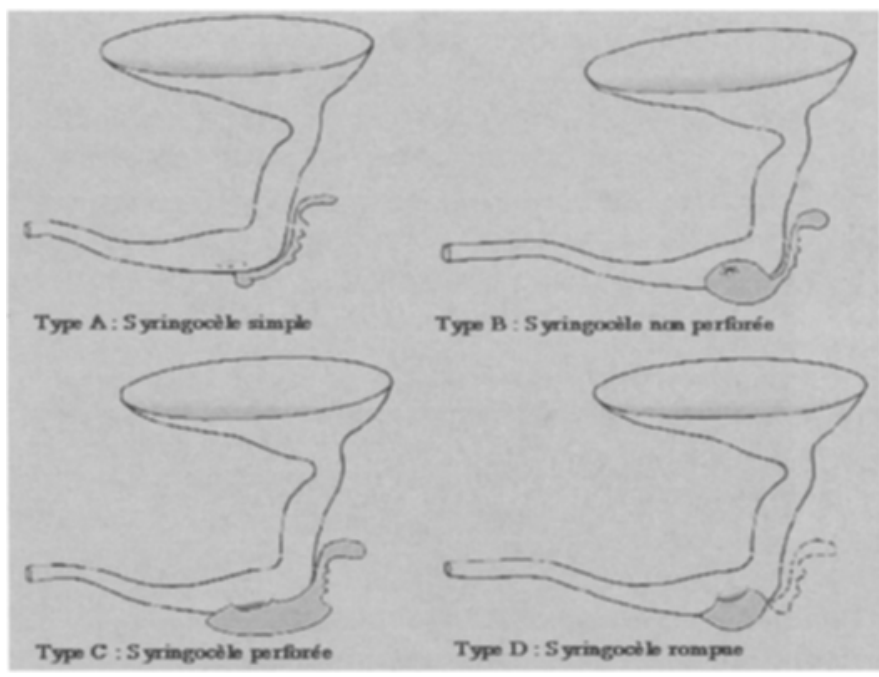

Figure 8 : Classification des syringocèles selon MAIZELS et al [9].

recommendation for management. J. Urol., 1988, $139: 87$ 88.

14. SALINAS SANCHEZ A.S., SEGURA MARTIN M., LORENZO ROMERO J., HERNANDEZ MILLAN I., RUIZ MONDEJAR R., VIRSEDA RODRIGUEZ J.A. : Ruptured syringocele of the Cowper's gland. Report of a case. Actas Urol. Esp., 1998, 22: 712-715

15. SHAW M.B., COLE O., TERRY T.R. : Cowper's syringocele causing painful haematuria in an adult. Sci. World J., 2004, $7: 308-310$

16. ZUGOR V., SCHROTT K.M., SCHOTT G.E. : Cowper's syringocele - a rare malformation of the male urethra. Erlangen experience in two example cases. Urol. Int., 2006, 76 : 182185.

Manuscrit reçu : avril 2007 ; accepté juin 2007.

\section{ABSTRACT}

Syringocele revealed by perineal abscess Kamel CHABCHOUB, Hussein KANDIL, Firas ZAHWA,
Philippe DANJOU

Purpose : The authors present a clinical report of a perforate syringocele diagnosed in a male patient and they review the clinical, radiological and therapeutic aspects of this disease.

Case report : A 50-year-old man attended the emergency room with perineal abscess. Computerized Tomography (CT) scan was used to establish the diagnosis. This 
patient also underwent urethrocystoscopy, sonography and retrograde urethrography.

Results : Treatment consisted of open surgical evacuation of the perineal abscess with bladder catheter. Urethrocystoscopy showed a large perforate syringocele in the urethra with no need for endoscopy marsupialization. No recurrence was observed at followup at 3 months.

Conclusion : Syringocele is a rare malformation which usually is asymptomatic. It can be congenital or acquired and can be classified into four morphological types. The most frequent treatment is endoscopic marsupialization of the syringocele. Open surgical excision is recommended for complicated syringocele with abscess or perineal pain.

Key-Words: bulbourethral glands, syringocele, abscess 